ACTA UNIVERSITATIS LODZIENSIS

Folia Litteraria Romanica 16, 2021

https://doi.org/10.18778/1505-9065.16.09

\author{
Gueorgui Armianov \\ CREE, INALCO \\ Université Sorbonne Paris Cité \\ (iD) https://orcid.org/0000-0001-8870-4616 \\ gueorgui.armianov@inalco.fr
}

\title{
L'amour et la sexualité dans les variétés non-standard bulgares
}

\begin{abstract}
RÉSUMÉ
L'article propose un regard critique sur le vocabulaire lié à l'amour, à l'acte sexuel, aux personnes impliquées dans des relations amoureuses, aux étapes et aux rôles dans le jeu de l'amour. L'analyse porte sur une période de plus de 100 ans - de la fin du $19^{\mathrm{e}}$ siècle jusqu'à nos jours en passant par l'époque du régime communiste. Pendant cette période, le vocabulaire spécifique argotique et familier lié à l'amour et au sexe a subi d'importants changements, tant au regard de la richesse des mots et de leurs significations, qu'au regard de l'origine, de la diffusion et de l'utilisation de ces unités lexicales. L'emploi dans le cinéma, la littérature, les chansons populaires, les médias et même dans le discours politique officiel des termes argotiques est aussi étudié. Une attention particulière est accordée à la désargotisation de plusieurs lexèmes et à leur intégration progressive dans le langage familier et la pratique langagière de personnes de tous les âges, professions et statuts sociaux.
\end{abstract}

MOTS-CLÉS - langue standard, dialectes, argot, langage familier.

\section{Love and Sexuality in Non-standard Bulgarian Language Varieties}

\section{SUMMARY}

This article offers a critical view at the vocabulary related to love, sex, people involved in romantic relationships and their roles in love-play. The analysis covers a period of more than hundred years - from the end of the nineteenth century, through the years of the communist regime to the present day. During this period, the specific slang and colloquial vocabularies related to love and to sex have undergone significant changes, both in terms of the abundance of words and their meanings, as well as in terms of origin, diffusion and use of the lexical units. Their function in cinema, literature, popular songs, the media and even in the official political discourse is also analysed. Particular attention is paid to the "unslangisation" of a number of lexemes and their gradual integration into colloquial speech and into the language practice of people of all ages, professions and social statuses.

KEYWORDS - standard language, slang, dialects, colloquial speech. 


\section{Introduction}

Dans les années qui ont suivi la libération de la Bulgarie de la domination ottomane en 1878 et au début du $20^{\mathrm{e}}$ siècle, plusieurs articles scientifiques sont parus, consacrés essentiellement aux argots professionnels et aux sociolectes lycéens. Cependant, ces variétés linguistiques n'ont pas trouvé de place dans la littérature ni dans la presse, même si leur usage était bien répandu, notamment dans les grandes villes. Plus tard, pendant les premières décennies du régime communiste (1944-1989), l'argot a été même interdit et ses usagers étaient souvent harcelés, voire poursuivis par les autorités.

Ce n'est que dans les années 1970 et 1980, que plusieurs livres ont été publiés, parlant pour la première fois ouvertement de l'amour et du sexe, très souvent en argot ou en langage familier ${ }^{1}$. Les romans faisaient encore exception et l'argot était plutôt parsemé ici et là à l'intérieur du texte. Parallèlement, des œuvres cinématographiques qui brisaient ce tabou ont aussi été réalisés, comme les films Avantage de G. Djulgerov, Orchestre sans nom de L. Kirkov, Hier d'I. Andonov.

Ces œuvres, par leurs sujets actuels et leurs dialogues expressifs, ont attiré un public jeune, mais ont aussi suscité la désapprobation des autorités officielles et des critiques conformistes car ils ont levé le voile cachant un discours argotique au quotidien qui officiellement était présenté comme un vestige du passé capitaliste. En même temps, quelques linguistes ont aussi tourné leur attention vers l'argot des étudiants bulgares (Karastoïcheva 1988 ; Armianov 1989).

\section{Divisions temporelles et linguistiques}

Sous réserve d'une simplification recherchée pour mieux décrire la situation en Bulgarie, on peut définir deux lignes de séparation temporelle, qui exposent des changements importants dans la conception et l'attitude envers l'amour et le sexe dans la société bulgare :

- la première ligne se situe à la fin des années 1970, quand certains signes timides de dégel sont apparus dans la société communiste bulgare ;

- la seconde est liée à la chute du régime communiste en Bulgarie en 1989, qui a abouti à la libération de la langue des restrictions idéologiques et culturelles imposées.

Jusqu'à la première démarcation, l'amour était décrit avec un nombre assez limité de lexèmes, le plus souvent neutres et très vagues. Ce n'est pas que des mots plus spécifiques désignant les contacts intimes, les parties du corps humain et l'acte sexuel n'existaient pas, mais ils étaient quasiment bannis dans la littérature, les arts et les médias. Cette situation linguistique « stérile » peut être prouvée par un examen

\footnotetext{
${ }^{1}$ Voir, par exemple, les romans de Dontcho Tsontchev, Ilya Velchev, Kiril Topalov.
} 
des dictionnaires de la langue bulgare de cette époque où le vocabulaire lié à l'amour et aux relations sexuelles était réduit à environ une dizaine d'unités ${ }^{2}$.

Par conséquent, il n'est pas étonnant que les recherches sur l'expression linguistique de l'amour soient extrêmement rares dans la littérature scientifique bulgare et qu'elles n'existent que depuis quelques décennies. Il semble même qu'il existe toujours une tradition de considérer l'amour comme une chose extrêmement personnelle et qu'en parler ou qu'écrire ouvertement à son sujet est le signe d'une éducation et d'une culture insuffisantes. C'est quelque chose qu'il ne faut pas montrer aux autres, qui doit rester caché dans la famille et dans la maison. Il est également important de noter que dans les études peu nombreuses sur l'amour, les analyses sont plus littéraires, stylistiques, fonctionnelles que lexico-sémantiques, et que le vocabulaire en question est souvent défini comme amoureux, simplement parce qu'il est placé dans un sujet qui s'y rapport ou dans un contexte d'amour.

Il convient aussi de souligner que le vocabulaire bulgare lié à l'amour et au sexe est presque exclusivement d'origine nationale - les mots d'origine étrangère sont rares et sont associés à des parties intimes du corps, à la science ou à des concepts, perçus traditionnellement comme impolis ou insultants. Dans les deux premiers cas, les mots étrangers sont principalement d'origine latine ou française, alors que dans le troisième, ils sont associés à des influences linguistiques plus anciennes, principalement orientales.

\section{Vocabulaires standard, argotique et familier du bulgare}

Dans la littérature bulgare, l'amour entre un homme et une femme est habituellement présenté sous un angle poétique et romantique. Les mots spécifiques liés à l'amour et les relations sexuelles sont peu nombreux et très abstraits. Outre les cas de mariage arrangé entre les familles, les relations entre les jeunes amoureux passent par quelques phases fondamentales : tout d'abord, les jeunes gens се харѐсват 'se plaisent, commencent à éprouver de l'attrait l'un pour l'autre' (Figure 1), puis ils се влюбват/се залюбвват 'tombent amoureux', ils се любят 's'aiment' et enfin, avec la bénédiction des parents, се взѐмат (litt. 'se prennent') ou ils се събйpam (litt. 's'unissent'), c'est-à-dire 'se marient'.

La langue standard ne nous fournit que deux substantifs qui expriment le sentiment : любо̀в et о̆бич, traduits en français de la même manière, par le mot 'amour'. Or, il existe une nette différence sémantique et connotative entre ces deux mots : любо̀в est un mot neutre qui exprime l'inclinaison de caractère passionnel envers une personne et qui peut être utilisé dans un large éventail

\footnotetext{
${ }^{2}$ Voir : Dictionnaire de la langue littéraire bulgare (Romanski, 1955), les trois premières éditions du Dictionnaire raisonné de la langue bulgare (Andrejchin, 1955, 1963, 1973), ainsi que les premiers volumes du Dictionnaire de la langue bulgare (Tcholakova, 1977).
} 
d'œuvres - littéraires, psychologiques ou sociologiques. Le substantif òбuч, par contre, est beaucoup plus intime, affectif et avec ce mot on dénomme l'amour tendre et concret entre deux personnes, ainsi que l'amour entre parents et enfants ou entre des gens proches.

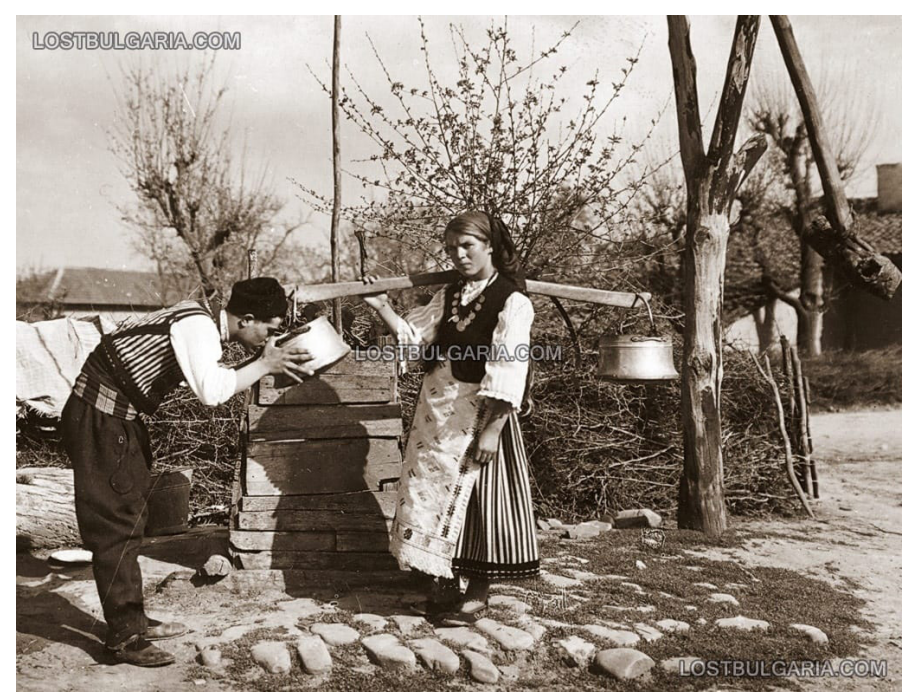

Figure 1. L'amour traditionnel en Bulgarie : les amants au puits, région de Yambol, c. 1920 ; source : www.LostBulgaria.com ; Reproduit avec l'accord écrit du responsable du site " Lost Bulgaria », Monsieur Peyo Kolev, envoyé à l'auteur le 25 septembre 2020.

La littérature nous présente plus de mots pour désigner les amoureux, tels que :

- substantifs : любо̀вник, любо̀вница (stand.) 'amant(e)', любовчѝя/ любовчййка (stand.) 'personne qui entre souvent dans des relations amoureuses ; coureur/coureuse', лйбе (folk. du verbe любя 'aimer'), изго̀ра (folk. du verbe изга̀рям 'brûler') et сѐвда (folk., de l'arabe sawda 'mélancolie', phonétiquement associée au mot turc sevgi 'amour') 'la personne dont on est amoureux'. Il faut noter que les trois derniers sont aujourd'hui considérés comme archaïques (Andrejchin, $2012: 295,416,873$ );

- adjectifs : влюेбен/влюббена 'amoureux / amoureuse', любѝм/любѝма 'bien-aimé(e)', лю̀бещ/лю̀беща 'aimant(e)' (en réalité, participes présents actifs), любо̀вен 'lié à l'amour', tous standard.

Dans le même champ sémantique, il existe aujourd'hui un seul verbe neutre : обйчам 'aimer', mais dans la littérature classique du $19^{\mathrm{e}}-20^{\mathrm{e}}$ siècles, on peut découvrir son synonyme poétique люेбя .

Cette signification classique est devenue archaïque et, dans la langue standard moderne, le verbe люेбя, le plus souvent à la forme réfléchie ou réciproque sert à exprimer le rapport sexuel : 'faire l'amour'. Il faut aussi souligner que 
le verbe neutre обйчам est peu productif, alors que son homologue rare et archaïque люेбя montre un spectre plus riche de verbes dérivés, par exemple : влюбвам се 'tomber amoureux', залюेвам 'commencer à aimer', разлюбввам 'cesser d'aimer', недолюेввам 'n'aimer pas trop'. Les deux verbes, обѝчам et люेбя, qu'il s'agisse d'une attitude émotionnelle ou d'une action physique envers quelqu'un, sont totalement dépourvus de connotations négatives. La signification moderne de люेб $я^{2}$ 'avoir une relation sexuelle' est, par contre, grammaticalement improductive.

On relève également les verbes standard уха̀жвам et задйрям 'faire la cour', ainsi que les expressions пра̀вя любо̀в/секс 'faire l'amour'. Les tabous culturels et sociaux ont obligé les gens à recourir à d'autres moyens pour exprimer des mots dits « interdits ». Par exemple, pour décrire l'acte sexuel les formules euphémiques utilisées sont : отѝшъл при нѐя 'il est allé chez elle', подчинѝл я на жела̀нието cu 'il l'a soumis à son désir', изпълнил съпружеските си задължения 'il a rempli ses devoirs conjugaux'.

Les variétés non standard du bulgare, comme l'argot ou le langage familier, nous dévoilent une image beaucoup plus variée et colorée par rapport à la langue standard. Traditionnellement, lorsqu'on parle de l'amour et du sexe en termes plus libres, décontractés, quelques mots très répandus viennent à l'esprit. En premier lieu, les locuteurs citent le verbe qui exprime l'acte physique de l'amour, puis passent par les termes utilisés pour les amants, les noms spécifiques des garçons et des filles tombés amoureux. Pourtant, ces termes figurent rarement dans les dictionnaires monolingues et bilingues : le verbe populaire eбà 'baiser' est inclus pour la première fois en 2012 dans la quatrième édition du Dictionnaire raisonné de la langue bulgare (Andrejchin, 2012 : 204) et seulement dans la version en ligne du Dictionnaire de la langue bulgare ; le mot argotique et familier pour 'petite amie' гадже ne figure pas dans les volumes publiés du Dictionnaire de la langue bulgare, même s'il a été ajouté récemment dans la version en ligne. De plus, leur registre linguistique ne fait pas l'unanimité : pour certains, ces termes sont argotiques, pour d'autres familiers ou populaires.

Quelle est la situation dans les variétés linguistiques non standard de la langue bulgare, en particulier dans l'argot des jeunes ? Parallèlement à l'amitié, la nourriture, les boissons et l'action de boire, le thème de l'amour et des relations sexuelles est l'un des plus importants dans la vie des jeunes. Si nous y ajoutons le thème des parties intimes du corps humain, qui est inextricablement lié à l'amour et déchaîne les fantasmes juvéniles, nous obtiendrons une couche lexicale très impressionnante. C'est pourquoi il est vraiment surprenant que dans ce champ sémantique nous ne trouvions pas de verbes argotiques, familiers ou populaires synonymes du verbe standard обѝчам 'aimer'. De tels verbes ne figurent pas dans le Dictionnaire de l'argot bulgare (Armianov, 2012).

Par contre, on découvre de nombreux verbes qui décrivent les phases bien distinctes des relations amoureuses : l'attrait pour quelqu'un, la drague, les 
rencontres amoureuses, les relations sexuelles. Ainsi, pour exprimer la première phase, le fait qu'ils sont attirés par quelqu'un, les jeunes Bulgares diront en argot qu'ils залйmam 'se toquent de quelqu'un, ont un penchant pour quelqu'un', qu'ils брӧйкат 'repèrent quelqu'un', грѝзкат 'rongent' ou кльо̀nат 'mangent, bouffent' quelqu'un'. Dans le discours familier, on remarque aussi des verbes et des expressions pittoresques, tels que : те сахльтнали litt. 'ils se sont amourachés l'un de l'autre', nàdaт си едйн по дру̀z 'ils ont le béguin l'un pour l'autre', очѝme им оста̀ват по някого 'leurs yeux restent attachés sur quelqu'un', хвърлят окоे на някого 'ils jettent les yeux sur quelqu'un' (Andrejčhin, 2012 : 563).

Le verbe argotique principal utilisé pour exprimer la deuxième phase, celle de la drague, est сва̀лям, littéralement 'descendre' qui d'un verbe purement argotique dans les années 1960-1980 est devenu progressivement un verbe familier qui ne fait aujourd'hui plus l'objet d'interdictions ou de restrictions lorsqu'il est utilisé dans un environnement informel. Le verbe est entré dans le cinéma, la littérature, les chansons et la presse quotidienne en Bulgarie. Par exemple, dans la chanson Seul au comptoir du bar, le chanteur de rock bulgare Georgi Minchev se demande :

Защчо ли да не взема тази нощ да се прежаля,

ето, гаджето отсреща продължава да ме сваля...

'Pourquoi, alors, ne pas sauter le pas cette nuit, voici, la nana d'en face continue de me draguer...'

Parmi les synonymes argotiques et familiers du verbe сва̀лям 'descendre', on peut noter бръмча̀ 'bourdonner', кръжа̀ 'tourner autour, graviter autour de quelqu'un' (comme un oiseau ou comme un avion), мрѐжa litt. 'faire un filet à quelqu'un', пикѝрам 'faire un piqué', зана̀сям се (fam.) 'baratiner'.

Dans la troisième phase, quand ils ont déjà réussi à tisser des liens avec quelqu'un, les jeunes, surtout les garçons, partagent avec fierté le fait qu'ils ont забъррсали litt. 'frôlé', забѝли 'enfoncé', заловѝли 'capturé', закова̀ли 'cloué', забо̀ли 'épinglé' une fille. Les exemples cités montrent le point de vue masculin des expressions, ce qui reflète entièrement les rôles de l'homme et de la femme dans une société traditionnelle. Toutefois, il faut souligner que certains de ces mots sont entrés dans le discours des filles et des femmes qui les utilisent sans crainte ni gêne.

Le vocabulaire argotique des jeunes Bulgares exprimant l'acte sexuel est particulièrement riche : dans le Dictionnaire de l'argot bulgare déjà cité, l'on trouve plus de quatre-vingts verbes et expressions verbales consacrés au sexe. Certains de ces verbes argotiques sont probablement sur le point de franchir la frontière avec le langage familier alors que d'autres sont sortis de l'usage avec

${ }^{3}$ Les exemples argotiques ont été pris du Dictionnaire de l'argot bulgare (Armianov 2012), ainsi que de la presse bulgare et des plusieurs pages de discussion sur Internet. 
le changement des générations ou en raison d'un emploi excessif et d'une usure graduelle.

Le verbe le plus connu est чу̀кам 'baiser' (litt. 'frapper, taper'), qui, à la voix active est utilisé exclusivement par les garçons et les hommes, mais qui dans sa forme pronominale, réciproque, чу̀кам се, est utilisé aujourd'hui aussi régulièrement par les garçons que par les filles. De plus, dans le cas du verbe pronominal, on observe un virage sémantique marqué de 'baiser quelqu'un' à 'avoir un rapport sexuel', par exemple : Тя̀ се чу̀ка о̀ще от гилна̀зията. 'elle a des rapports sexuels depuis le lycée'. Parmi les autres verbes, les plus frappants, qui illustrent la pensée imaginative et métaphorique des usagers de l'argot d'étudiants on trouve : дру̀сам 'secouer', забйвам пироेнче 'enfoncer un clou', ка̀ивам 'atterrir', люлѐя 'bercer', опра̀мвам 'polliniser', хвърлям хайвѐр 'jeter son frai', чаршафо̀свам се 's' emballer dans les draps', шиия 'coudre', шла̀йфам 'raboter', иля̀nам 'patauger'.

Les participants à une relation amoureuse ont aussi leurs noms spécifiques, qui, selon la situation, peuvent présenter certaines nuances et être chargés d'une connotation positive ou négative. En règle générale, pour dénommer le garçon, l'argot bulgare actuel utilise un vocabulaire machiste de caractère mélioratif. Le garçon est un бòu de l'anglais 'boy', броя̀ч 'un compteur' puisqu'il compte ses conquêtes, львьче 'un lionceau', пич 'une personne attractive, sympa, un vrai mec' du mot turc piç 'rejeton, bâtard', тъпка̀ч 'quelqu'un qui farcit, qui remplit' ou unàza 'une épée'. Le langage familier nous offre плѐйбой de l'anglais 'playboy', мàyo de l'espagnol 'macho', коика̀р 'dragueur'.

Pour décrire une jeune fille ou une femme, rien que dans l'argot on trouve plus de cent mots et expressions, comme : ка̀ка litt. 'grande sœur', стъкларйя 'verrière', стъълен бага̀ж 'bagage en verre', ча̀йка 'mouette, га̀dже 'bébé, nana' de la forme au pluriel du mot romani gadjo 'étranger, personne non tsigane', гърла de l'anglais girl, etc. Toutefois, la plupart des mots sont créés en fonction de l'apparence physique de la jeune fille :

- une fille jolie, attirante : а̀zнешко 'agneau', бедро̀ 'cuisse', бонбо̀н(че) '(petit) bonbon', броійлер 'poulet élevé pour de la viande' du même mot anglais 'broïler', джу̀фка 'nœud de ruban', мàue et мàuка 'chaton', парчè 'morceau (de viande)'; бъбрече 'petit rognon';

- une fille expérimentée qui a de nombreux contacts sexuels : aкỳлa 'requin', бургйя 'mèche, foret à percer, гъзйй 'fille avec une grosse fesse', кара̀чка 'conductrice', цеепеня̀к 'fendeuse' (mais aussi fille appétissante) ;

- une fille moche, laide : вампйp 'vampire', годзѝла 'Godzilla', кобѝла 'jument', коेбра 'cobra', кра̀ва 'vache', крокодѝл 'crocodile', чапла 'héron', скумрйя litt. 'maquereau';

- une fille grosse : бỳua 'grosse motte', гỳda 'cochon ; grande bille de jeu', гюлѐ 'boulet de canon';

- une fille stupide : коко̀шка 'poule', па̀тка 'canard', гъскка 'оіе', ку̀кувийа 'coucou', овиа̀ 'brebis', кѝфла 'croissant brioché'. 
Comme on pouvait s'y attendre, les rapports sexuels mènent souvent à la grossesse, pour laquelle le langage familier et l'argot des jeunes Bulgares possèdent plusieurs mots et d'expressions colorés, pittoresques qui dans la plupart des cas ne sont ni corrects, ni agréables. Pour les garçons et les hommes, on dit qu'ils наду̇ват, напо̀мпват 'gonflent' une fille ; pour une fille ou une femme on déclare qu'elle е гльттнала то̀пката 'a avalé le ballon' ou е наду̀ла бало̀на (то̀кката) 'a gonflé le ballon (la balle)', c'est-à-dire qu'elle est enceinte, ou qu'elle е връзала с детѐ ня̀кого 'a attaché quelqu'un avec un enfant'. Cependant, il est important de souligner que ces mots et expressions ne visent pas à blesser les femmes, mais plutôt à plaisanter avec elles et à mettre en évidence la masculinité, la virilité des garçons.

Nous trouvons également de nombreux mots et expressions pour décrire les poses et les activités sexuelles. Pour l'acte sexuel traditionnel dans l'argot des étudiants, nous trouvons des substantifs tels que : uгpà в nàpmep 'jeu à terre' (terme de la lutte gréco-romaine), кафѐ с ра̀йбер 'café à loquet', крева̀тна гимна̀стика 'gymnastique au lit', etc. Pour l'acte de fellation, on découvre des mots comme : вувузѐла 'vuvuzela', свйрка 'sifflet' ainsi que des verbes et expressions comme духам 'siffler', ма̀лка музика̀лна фо̀рма 'petite forme musicale', пра̀вя свйрки 'faire des sifflets', свйря на кава̀л (кларинѐm, дудук) 'jouer de la flûte, de la clarinette, du pipeau', certain(e)s très grossièr(e)s et vulgaires.

\section{Conclusions}

Le fait que ce vocabulaire argotique est résolument sexiste, voire misogyne frappe immédiatement l'esprit. Dans l'argot des jeunes, c'est le garçon, l'homme qui est le personnage actif dans la dénomination de la partenaire et des actes liés à l'amour. Toutefois, il faut préciser que depuis quelques décennies, on est témoin d'une émancipation linguistique évidente et que de plus en plus les jeunes filles, les femmes assument ce rôle actif. Cela se reflète dans leur discours, dans les mots qu'elles se sont approprié du langage des garçons et qui font désormais partie de leur vocabulaire à elles.

Il serait naïf de penser que la langue standard créerait dorénavant des mots neutres qui combleraient les lacunes lexicales évoquées. Par conséquent, la solution doit plutôt se trouver dans les confins du langage familier et des sociolectes modernes. Au fil des ans, il a été constaté qu'en raison de leur fort caractère expressif certains mots et expressions abandonnaient leur environnement naturel et commençaient à circuler dans d'autres variétés non standard plus larges. Plusieurs argotismes ont subi une désargotisation totale et sont aujourd'hui considérés comme appartenant au vocabulaire expressif familier. Ils sont entrés dans le discours de personnes de tous âges, professions, statuts sociaux, culture et éducation et ont trouvé une place dans les dictionnaires. On peut spéculer qu'un 
jour, après avoir complètement perdu leur caractère moqueur ou grossier, ces mots franchiront la frontière et deviendront une partie intégrante de la langue standard bulgare.

\section{Bibliographie}

ANDREJCHIN, Lubomir et al. (2012), Bălgarski tălkoven rečnik (4éd.), Sofia, Nauka i izkustvo ARMIANOV, Gueorgui (1989), Zargonăt, bez kojto (ne) možem, Sofia, Nauka i izkustvo ARMIANOV, Gueorgui (2012), Rečnik na bălgarskija žargon, Sofia, Figura KARASTOÏČEVA, Tsvetana (1988), Bălgarskijat mladežki govor, Sofia, Nauka i izkustvo ROMANSKI, Stoyan et al. (1955), Rečnik na bălgarskija knižoven ezik, Sofia, Académie bulgare des sciences.

TCHOLAKOVA, Kristalina et al. (éds) (1977-2015), Rečnik na bălgarskija ezik, vol. 1-15, Sofia, Académie bulgare de sciences.

Gueorgui Armianov - maître de conférences en linguistique bulgare à l'Institut national des langues et civilisations orientales, Paris. Il a été chargé de cours en linguistique bulgare à l'Université de Sofia, chercheur à l'Académie bulgare des sciences et lecteur de langue, littérature et civilisation bulgares à l'Université de Londres, l'Université d'Oxford (1992-1994) et à l'Université de Strasbourg (19982005). Ses intérêts scientifiques portent sur la grammaire, la lexicologie et la lexicographie bulgares, la sociolinguistique, les variétés non standard. 